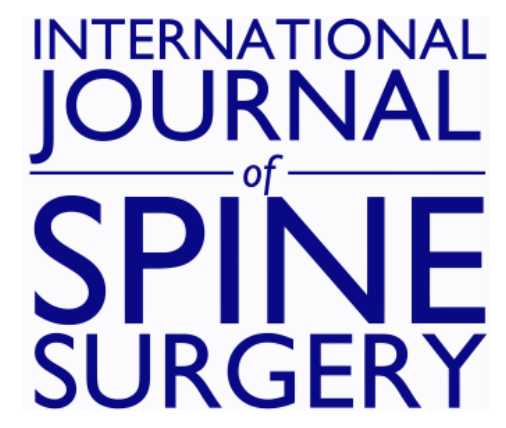

\title{
Teardrop fracture following head-first impact in an ice hockey player: Case report and analysis of injury mechanisms
}

James J. Yue, Paul C. Ivancic and David L. Scott

Int J Spine Surg 2016, 10 ()

doi: https://doi.org/10.14444/3009

http://ijssurgery.com/content/10/9

This information is current as of April 26, 2023.

Email Alerts Receive free email-alerts when new articles cite this article. Sign up at:

http://ijssurgery.com/alerts

The International Journal of Sdoing Surgehth: 2397 Waterbury Circle, Suite 1,

Aurora, IL 60504, Phone: +1-630-375-1432

(c) 2016 ISASS. All Rights Reserved. 


\section{Teardrop fracture following head-first impact in an ice hockey player: Case report and analysis of injury mechanisms}

James J. Yue, MD, Paul C. Ivancic, PhD, David L. Scott, MD, PhD, DVM

Department of Orthopaedics and Rehabilitation, Yale University School of Medicine, New Haven, Connecticut, USA

\section{Abstract}

Background

We report a case of a young male athlete who sustained a three column displaced teardrop fracture of the C5 vertebra due to a head-first impact in hockey, suffered neurapraxia, yet made full neurological recovery. This full recovery was in sharp contrast to multiple case series which reported permanent quadriplegia in the vast majority of teardrop fracture patients. We investigate the etiology and biomechanical mechanisms of injury.

\section{Methods}

Admission imaging revealed the teardrop fracture which consisted of: a frontal plane fracture which separated an anterior quadrilateral-shaped fragment from the posterior vertebral body; a vertical fracture of the posterior vertebral body in the sagittal plane; and incomplete fractures of the neural arch that initiated superiorly at the anterior aspect of the spinous process and left lamina adjacent to the superior facet. Epidural hematoma in the region of the C5 vertebra was observed in addition to disc and ligamentous disruptions at C4-5 and C5-6. Our patient was ultimately treated surgically with anterior fusion from $\mathrm{C} 4$ through $\mathrm{C} 6$ and subsequently with bilateral posterior fusion at C5-6.

Results

The injuries were caused by high-energy axial compression with the neck in a pre-flexed posture. The first fracture event consisted of the anterior vertebral body fragment being sheared off of the posterior fragment under the compression load due in part to the sagittal plane concavity of the C5 inferior endplate. The etiology of the vertical fracture of the posterior vertebral body fragment in the sagittal plane was consistent with a previously described hypothesis of the mechanistic injury events. First, the C4-5 disc height decreased under load which increased its hoop stress. Next, this increased hoop stress transferred lateral forces to the C5 uncinate processes which caused their outward expansion. Finally, the outward expansion of the uncinate processes caused the left and right sides of the vertebral body to split and spread. Evidence in support of this mechanistic event sequence was provided by the neural arch fractures which initiated superiorly, average angulation of the C5 uncinate processes, and similar wellestablished mechanisms causing vertical fractures at other spinal regions.

\section{Conclusions}

Our case study and analyses provide insight into the etiology of the specific teardrop fracture patterns observed clinically.

KEYWORDS: TEARDROP FRACTURE, INJURY MECHANISM, CERVICAL SPINE, HOCKEY, IMPACT BIOMECHANICS

VOLUME 10 ARTICLE 9 DOI: $10.14444 / 3009$

\section{Introduction}

Teardrop fracture is the most severe ${ }^{1}$ and unstable injury of the cervical spine resulting in permanent quadriplegia in the vast majority of cases $^{2-4}$ and even death. ${ }^{5}$ The injury has been well described clinically: forward displacement of an anterior fracture fragment of the vertebral body (VB); midsagittal fracture of the posterior VB fragment with retropulsion causing cord compression and neurological sequelae; one or more neural arch fractures; widening of the interlaminar spaces, interspinous spaces, and facet joints; and ligamentous disruption at the intervertebral level inferior to the affected vertebra. ${ }^{2,4,6}$ It occurs most often at $\mathrm{C} 5$, followed by $\mathrm{C} 6$, and $\mathrm{C} 4$, and has been reported at multiple adjacent vertebrae..$^{4-6}$ The upper 
cervical column, consisting of the vertebrae at and above the site of injury, is displaced posteriorly relative to the lower column. ${ }^{6}$ The anterior fracture fragment with its triangular or quadrilateral shape ${ }^{4}$ is thought to resemble a tear dripping from the VB, indicative of the negative neurological outcome often associated with the injury.

Teardrop fracture is caused by high-energy axial compression of the flexed cervical spine due to trauma involving head impact such as: diving into shallow water, fall from high height, motor vehicle crash, or sports impact. ${ }^{2,7}$ Teardrop fractures have been described due to spear tackling in football ${ }^{2}$ in which the player flexes their head and neck which straightens cervical lordosis and strikes the opponent with the top of their helmet. Spinal cord injuries in ice hockey are most commonly reported due to head-first impact into either the boards or an opponent. ${ }^{8,9}$ In these trauma scenarios, the cervical spine sustains excessive transient compression causing fractures due to abrupt deceleration of the helmeted head and oncoming momentum of the torso mass. While the global mechanistic events are well understood, less is known regarding the etiology of the specific teardrop fracture patterns observed clinically.

Teardrop fracture etiology is dependent in part upon vertebral anatomy. A VB of the middle or lower cervical spine has distinct features: 1) a superior surface with uncinate processes that project upward from its lateral margins and 2) a saddle-shaped inferior surface ${ }^{10}$ convex laterally and concave anteroposteriorly with the anteroinferior corner often skewed forward. ${ }^{11,12}$ Bilateral Luschka joints, between the uncinate processes and the inferior borders of the adjacent VB, have been shown in mathematical modeling to increase cervical motions, while the uncinate processes reduce motions and coupling. ${ }^{13}$

Hypotheses regarding teardrop fracture etiology, outlined by Kazarian et al. ${ }^{12}$ are based upon radiographic analyses of cervical trauma patients, biomechanics, and the observations of vertebral anatomy. The anterior fracture fragment forming the teardrop is hypothesized to have been sheared off of the larger posterior VB fragment. ${ }^{6}$ This is due to its forced compression between the adjacent bodies in a pre- flexed posture with the fracture line of the "teardrop" related in part to the sagittal plane concavity of the inferior endplate. Continued compressive load combined with flexion causes retropulsion of the posterior VB fragment resulting in cord compression injury.

Several hypotheses exist regarding the etiology of the vertical VB fracture in the sagittal plane caused by the compressive load. Some believe that the superior nucleus is forcibly wedged into the superior endplate causing it to split and spread. ${ }^{14}$ Another hypothesis is that the fracture may initiate and propagate at the basivertebral veins. ${ }^{12}$ Vertebral arterial anatomy indicates that the largest vein enters the posterior surface of the VB in the midsagittal plane and penetrates to approximately half its anteroposterior width. ${ }^{15}$ Lastly, the compression load may decrease the height of the superior disc and increase its hoop tension which transfers lateral forces to the uncinate processes causing their outward expansion. ${ }^{12}$ The lateral forces which separate the right and left sides of the VB often cause associated fractures of the neural arch.

The purpose of the present study was to report a case of an athlete who suffered neurapraxia, yet made full neurological recovery, after sustaining cervical teardrop fracture due to a head-first impact in ice hockey. We investigated the etiology and biomechanical mechanisms of injury based upon the injury causing environment, patient characteristics, review of the patient's medical records and diagnostic images, and syntheses of prior case series and biomechanical studies.

\section{Case Report}

\section{Injury Causation}

Video footage of the accident was not available. A 17-year old male athlete participating in a fall league ice hockey game reported that he and an opponent, who had the puck, skated towards each other at high speed. The athlete tripped over a teammate's stick, fell forward, and the top of his helmet forcefully impacted the opponent's leg at approximately midthigh. At the time of impact, his neck was straight and his stick was held in front of him with both hands. His stick broke upon contact with the oppo- 
nent, likely the lower leg, at approximately the same time the head impact occurred. He was unable to get up and reported feeling pins and needles in his hands and upper arms. The patient reported approximately 1 minute of functional quadriplegia. Neck pain after the impact was minimal. He had transient quadriplegia until external stabilization and distraction could be applied with a well fitting halo-vest. His leg function returned prior to arm function.

\section{Clinical Imaging, Diagnosis, and Treatment}

Upon arrival at the emergency room of an off-site facility, the patient experienced neck pain and transient quadriparesis. He had weakness of his upper extremities mainly in the triceps. Admission computed tomography (CT) scans performed at the off-site fa- cility revealed a teardrop fracture of the $\mathrm{C} 5$ vertebra (Figure 1). The upper cervical column, consisting of C5 and superior vertebrae, was displaced posteriorly relative to the lower column. The posteroinferior corner of the C5 VB was displaced $4.0 \mathrm{~mm}$ into the spinal canal (measurement made using Synapse 3.2.1, Fujifilm Medical Systems USA Inc., Stamford, CT, USA).

The sagittal plane CT sequence (Figure 1 A-C) demonstrated a quadrilateral-shaped fracture fragment of the anterior VB with fracture comminution of its superior and anterosuperior regions. This anterior fracture fragment was displaced forward and rotated in extension. Its anteroposterior width was 6.3 and $4.7 \mathrm{~mm}$ at its inferior and superior surfaces, re-

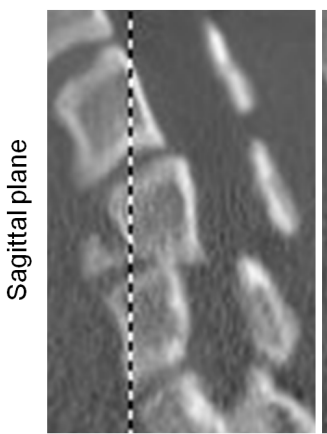

a) left

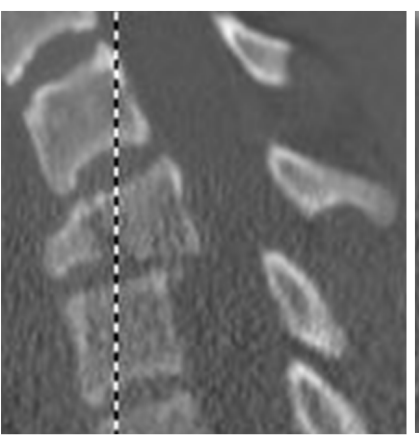

b) midsagittal

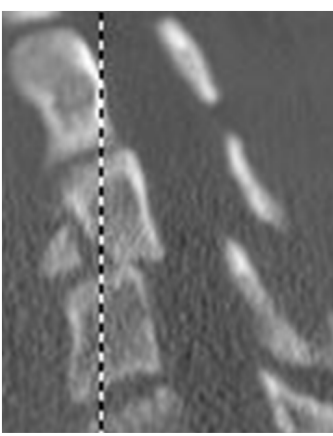

c) right

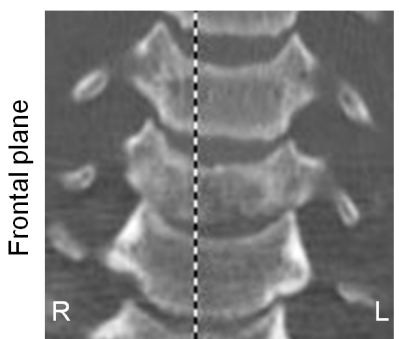

d) anterior

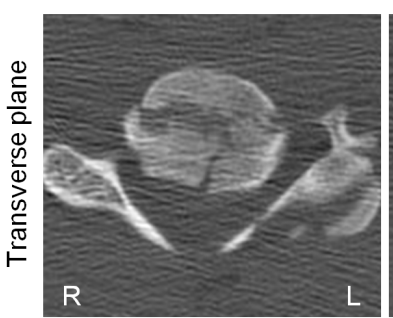

g) inferior

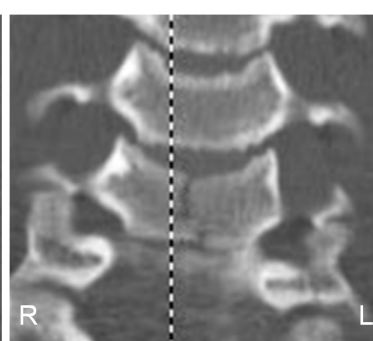

e) mid

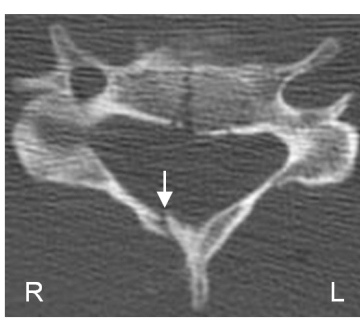

h) mid-inferior

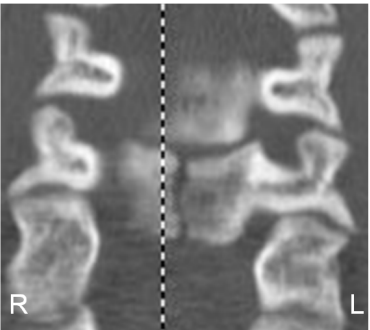

f) posterior

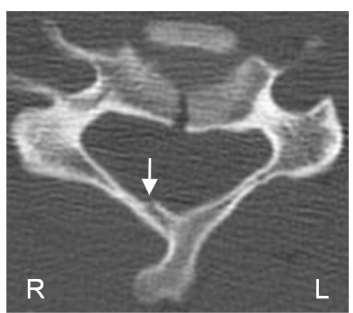

i) mid-superior

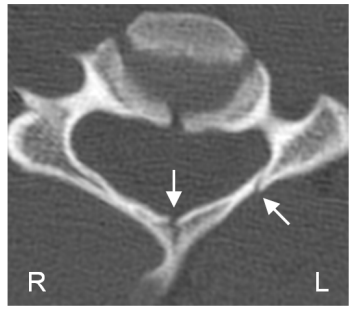

j) superior

Fig. 1. Admission CT scans demonstrating teardrop fracture of the $C 5$ vertebra. The sagittal plane sequence shows forward displacement and extension of the anterior VB fragment and retropulsion of the posterior VB fragment into the canal: a) left, b) midsagittal, and c) right. The frontal plane sequence shows the vertical fracture at the: $d$ ) anterior, e) mid, and f) posterior regions of the posterior VB fragment. The transverse plane sequence shows the aforementioned injuries in addition to the neural arch fractures indicated with arrows: $g$ ) inferior, h) mid-inferior, i) mid-superior, and j) superior. Note from the sagittal place sequence that the spine is tilted anteriorly relative to the axes of the scanner. Consequently, the anteroinferior region of the C4 VB appears in panels $i$ and $j$ of the transverse plane sequence. 
spectively, corresponding to $42 \%$ and $34 \%$ of the total VB width. A horizontal gap of $4.4 \mathrm{~mm}$ was measured inferiorly between the anterior and posterior VB fracture fragments in the midsagittal plane (Figure $1 \mathrm{~B}$ ).

The frontal plane CT sequence (Figure $1 \mathrm{D}-\mathrm{F}$ ) of the posterior VB fragment demonstrated its vertical fracture in the sagittal plane. The heights at the anterior (Figure $1 \mathrm{D}$ ) and middle (Figure $1 \mathrm{E}$ ) of this fracture fragment were $67 \%$ and $77 \%$ of the posterior cortex height (Figure $1 \mathrm{~F}$ ). At the posterior cortex (Figure 1 F), the vertical fracture was offset to the right of the midsagittal plane by $0.8 \mathrm{~mm}$ as it extended from the superior surface to the middle of the VB and shifted to the midsagittal plane at the inferior surface of the VB. This shift was more pronounced at the mid region (Figure $1 \mathrm{E}$ ).

The transverse plane CT sequence (Figure 1 G-J) demonstrated the aforementioned fractures in addition to neural arch fractures. Incomplete fractures that initiated superiorly were observed at the anterior aspect of the spinous process and left lamina adjacent to the superior facet (Figure $1 \mathrm{~J}$ ). The spinous process fracture was associated with an incomplete fracture of the right lamina observed at mid-height of the $\operatorname{arch}$ (Figure $1 \mathrm{H}, \mathrm{I}$ ).

Admission magnetic resonance imaging (MRI) performed at the off-site facility demonstrated the retropulsion of the upper cervical column with epidural hematoma in the region of the $\mathrm{C} 5$ vertebra (Figure 2). Disc and ligamentous disruptions were observed at C4-5 and C5-6.

The patient was transferred to our institution a day following the accident. Neurological examination indicated sensation was grossly present in the C5-S1 nerve distribution with subjective paraesethesias in the $\mathrm{C} 5, \mathrm{C} 6$, and $\mathrm{C} 7$ distribution. Halo-vest fixation was applied.

Anterior cervical fusion was performed 2 days after the accident. The halo-vest was removed and a cervical collar and tongs with $10 \mathrm{lbs}$ traction were applied which produced satisfactory spinal alignment. A left anterolateral approach to the cervical spine was used. Interspace distraction using Caspar pins did not re- veal significant facet separation at the site of injury. Discectomies were performed at C4-5 and C5-6 out to the Luschka joints. Central corpectomy was performed at $\mathrm{C} 5$ with removal of the posterior cortex and remaining posterior longitudinal ligament. A large fragment of posterior cortex was evident within the canal. A tricortical iliac crest bone graft was inserted and the traction weight removed. The graft was found to be very stable. Anterior plate fixation was applied at $\mathrm{C} 4$ through $\mathrm{C} 6$ (Figure $3 \mathrm{~A}, \mathrm{~B}$ ). The patient was able to move all extremities immediately following the operation. Nonunion was observed at 5 months at C5-6 (Figure $3 \mathrm{C}$ ) which was treated with bilateral posterior fusion (Figure $3 \mathrm{D}$ ).

\section{Discussion}

We report a case of a young male athlete who sustained cervical teardrop fracture due to a head-first impact in ice hockey, suffered neurapraxia, yet made full neurological recovery. Multiple patient-related and biomechanical factors contributed to his full neurological recovery, which is highly uncommon based upon prior case series of athletes who sustained similar injuries. ${ }^{2,8,9}$ Prior to the accident, our patient was in overall good health and good physical condition and did not have a history of smoking or usage of alcohol or illicit drugs. His ligaments at C5-6, although disrupted, had sufficient strength and viscoelasticity

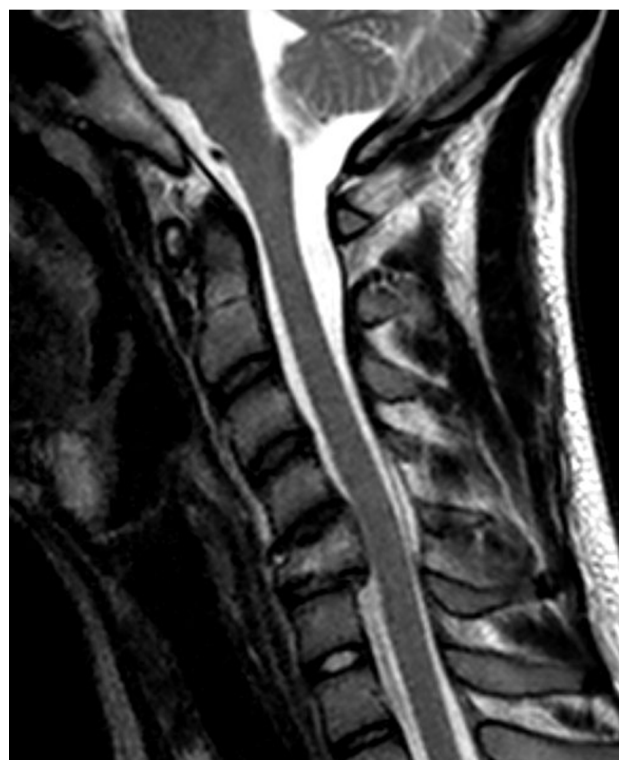

Fig. 2. Admission T2-weighted MRI of the cervical spine demonstrating posterior displacement of the upper cervical spinal column, at and above the C5 vertebra, with epidural hematoma noted in the region of C5. 
to limit the transient retropulsion of the C5 VB during impact and protect his cord from sustaining a permanent lesion.

We measured $4.0 \mathrm{~mm}$ midsagittal canal occlusion from the post-injury CT due to retropulsion of the C5 posterior VB fragment (Figure $1 \mathrm{~B}$ ). While the magnitude of transient canal occlusion during impact was not known, previous biomechanical studies suggest that it was significantly greater than that measured clinically from the post-injury radiographic images. ${ }^{16,17}$ The biomechanical studies indicate that the

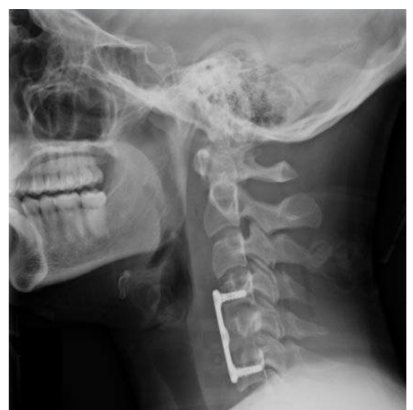

a) post-op: anterior fusion
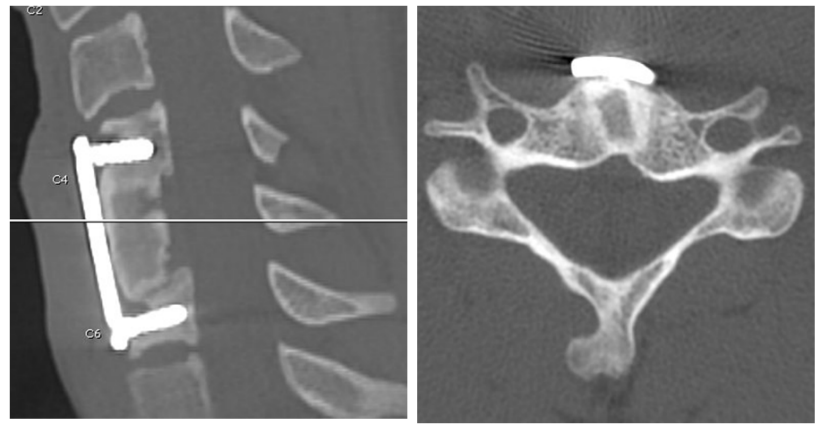

c) 5 months
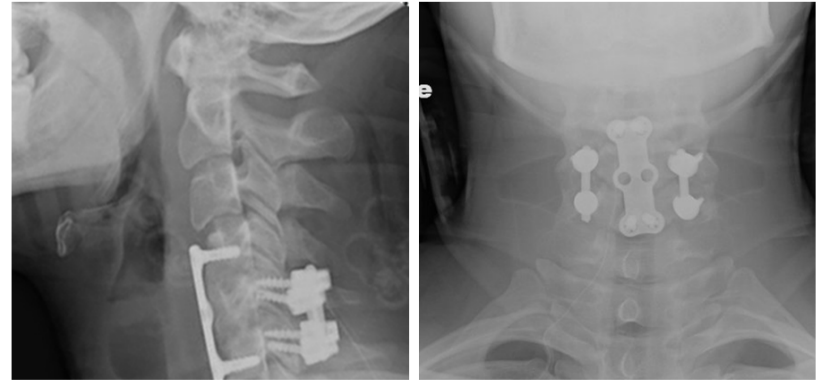

d) post-op: posterior fusion

Fig. 3. Lateral radiographs demonstrating the corrected spinal alignment and stability resulting from the anterior fusion at $\mathrm{C} 4$ through $\mathrm{C} 6$ with bone graft restoring height of the C5 VB: a) post-op and b) 3.5 months.

Nonunion at C5-6 was observed at 5 months (c). Bilateral posterior fusion was subsequently performed at C5-6 (d). peak transient neck loads can occur as early at 20 to $60 \mathrm{~ms}$ following head impact indicative of the onset of fracture initiation. ${ }^{18}$ Carter et al (2000) applied high-energy compressive loading to straightened cervical spine specimens causing burst and wedgecompression fractures and measured peak canal occlusion transiently during impact (72\% occlusion), immediately post-impact with the spine in recoil position (47\% occlusion), and subsequently post-injury from CT scans ( $22 \%$ occlusion). Considering these biomechanical data, our patient was very fortunate to not have sustained permanent neurological sequelae.

The admission CT and MR images of our patient (Figure 1 and Figure 2) may be used to deduce the biomechanical mechanism of injury and evaluate previous hypotheses regarding the etiology of the teardrop fracture patterns. The anterior VB fragment was quadrilateral shaped (Figure $1 \mathrm{~B}$ ) with fracture comminution of its superior and anterosuperior regions and did not include the uncinate processes. Its sagittal width was $42 \%$ and $34 \%$ of the total VB width at the inferior and superior surfaces, respectively, within the $13 \%$ to $75 \%$ range for this measurement reported among 52 football players who sustained cervical teardrop fracture. ${ }^{2}$ The anterior VB fragment of our patient had not sustained a vertical sagittal fracture indicating that its separation from the posterior VB fragment was the first fracture event due to the compressive load in a pre-flexed neck posture. Continued compression and flexion caused the anterior fragment to be displaced forward, rotated in extension, and partially crushed. The fracture line of the anterior fragment was related in part to the sagittal plane concavity of the inferior endplate, consistent with the hypotheses of Kim et al. ${ }^{6}$ and Kazarian et al. ${ }^{12}$

Following separation of the anterior VB fragment, the posterior VB fragment sustained a vertical fracture in the sagittal plane (Figure $1 \mathrm{D}-\mathrm{F}$ ). This vertical fracture was offset to the right of the midsagittal plane at its superior surface and propagated to the midsagittal plane at its inferior surface. The continued compression and flexion caused retropulsion of the posterior VB fragment with fracture comminution and reduced height at its anterior and mid regions (Figure 1 D, E). 
Kazarian et al. ${ }^{12}$ hypothesized that the transient compressive load causes the vertical fracture of the VB fragment in the sagittal plane due to the following sequence of mechanistic responses. The height of the disc superior to the teardrop fracture decreases under load which increases its hoop stress. This increase in hoop stress transfers lateral forces to the uncinate processes causing their outward expansion. Lastly, the outward expansion of the uncinate processes causes the left and right sides of the VB to split and spread. The mechanism of the vertical fracture of the posterior VB fragment in our case study supports the hypothesis proposed by Kazarian based upon the following factors which we address in detail below: 1) associated neural arch fractures, 2) average angulation of the $\mathrm{C} 5$ uncinate processes, and 3) similar well-established mechanisms causing vertical fractures at other spinal regions.

Associated neural arch fractures were observed at the superior surfaces of the anterior aspect of the spinous process and the left lamina directly adjacent to the superior facet with no associated fracture comminution (Figure $1 \mathrm{~J}$ ). The spinous process fracture extended into an incomplete fracture of the right lamina at the mid-height of the neural arch (Figure 1 $\mathrm{H}, \mathrm{I})$. These fracture patterns indicated fracture initiation at the superior surface of the neural arch which propagated inferiorly due to outward expansion of the attached posterior VB fragment. This suggests that the vertical fracture of the posterior VB fragment in the sagittal plane initiated at its superior surface and propagated inferiorly due to outward expansion of the $\mathrm{C} 5$ uncinate processes. Based upon the cross-sectional anatomy of the $\mathrm{C} 5$ neural arch, it is weakest at its laminae and anterior to the spinous process when bent in the transverse plane. The locations of the present arch fractures are consistent with those observed in previous studies of teardrop fracture patients. ${ }^{6,12,19}$

The fracture initiation at the superior endplate of the C5 posterior VB fragment with propagation inferiorly was most likely due to transfer of compressive load through the C4-5 Luschka joints combined with increased hoop stress of the C4-5 disc. The average inclination from horizontal of the $\mathrm{C} 5$ uncinate processes in the frontal plane is $55.5^{\circ}$ which is $4.2^{\circ}$ to $12.8^{\circ}$ larger than at adjacent vertebrae $\left(51.3^{\circ} \mathrm{C} 3 ; 50^{\circ} \mathrm{C} 4\right.$; 49. $2^{\circ} \mathrm{C} 6$; and $\left.42.7^{\circ} \mathrm{C} 7\right) .{ }^{20}$ The transient axial compressive load was transferred to radial force sufficient to split and spread the two sides of the $\mathrm{C} 5$ posterior VB fragment. Lateral displacement of the two sides of the VB fragment was likely larger transiently during injury as compared with that observed on the post-injury CT (Figure 1E, F). Multiple clinical studies have indicated that teardrop fracture occurs most often at C5, followed by C6, and C4. ${ }^{4-6}$ We hypothesize that the high incidence of teardrop fracture reported at $\mathrm{C} 5$ is due in part to the steeper inclination of its uncinate processes as compared with adjacent vertebrae. Assuming similar transient axial load throughout the subaxial cervical spine and similar mechanical properties of the discs, the $\mathrm{C} 5$ vertebra will sustain greater radial force due to the increased hoop stress of the C4-5 disc. This may predispose the $\mathrm{C} 5 \mathrm{VB}$ to sustaining greater lateral forces leading to a vertical fracture in the sagittal plane as compared to the adjacent vertebrae.

While the angulation of the uncinate processes in the frontal plane enables axial compressive force to be transferred to radial force, their average angulation in the transverse plane ${ }^{20}$ suggests an additional component of anterior or posterior shear force. This angulation measured in cadaveric studies would cause anterior shear at C3, C4, and C5 and posterior shear at the inferior cervical vertebrae. The average angulation in the transverse plane is smallest at $\mathrm{C} 5,7.3^{\circ}$, as compared to the adjacent vertebrae. ${ }^{20}$ Following separation of the anterior and posterior fracture fragments of the C5 VB in our case study, continued high-energy compression combined with flexion occurred. Any anterior shear of the $\mathrm{C} 5$ vertebra due to transfer of the compressive load through the uncinate processes would be minimal in comparison with the large posterior shear that caused the posterior VB fragment to be wedged into the canal (Figure 1 B).

Similar well-established mechanisms cause vertical vertebral fractures due to axial compression at other regions of the cervical spine. Jefferson ${ }^{21,22}$ described the transfer of axial load to radial force in the atlas due to the inclination of its superior and inferior facets, often resulting in fractures of the anterior 
arch, posterior arch, or both. This atlantal fracture mechanism is similar to that which caused the incomplete neural arch fractures associated with the C5 teardrop fracture in our case study. A difference is that radial force causing atlantal arch fractures initiates at both the superior and inferior facets of $\mathrm{C1}$ due to head-first impact, while the radial force that caused the vertical split of the C5 posterior VB in our case study initiated superiorly from its uncinate processes.

Our case study does not support the other hypotheses regarding the etiology of the sagittal VB fracture caused by the compressive load. Stimpfl (1949) suggested that the superior nucleus may be forcibly wedged into the superior endplate causing it to split and spread. We carefully reviewed the admission MRI of our patient and did not observe nucleus or annular fibers within the vertical VB fracture. Another hypothesis is that the fracture may initiate and propagate in coincidence with the basivertebral veins. ${ }^{12}$ While little data exists on quantitative arterial anatomy of the cervical VBs, Harris et al. ${ }^{15}$ observed that the largest vein enters the posterior surface of the VB in the midsagittal plane and penetrates to approximately half its anteroposterior width. Observations and measurements from their diagrammatic study indicate a maximum vein diameter on the order of $0.25 \mathrm{~mm}$. The fracture patterns of our case study suggest that the vertical VB fracture initiated from the superior surface of the VB and not from the basivertebral veins. However, our case study does not provide insight into whether the hollowed bone channels for the basivertebral veins influenced the propagation path of the vertical fracture.

Following the accident, our patient was treated operatively with anterior cervical fusion following discectomies at C4-5 and C5-6 and corpectomy and insertion of bone graft at $\mathrm{C} 5$. Following the discectomies, we did not observe significant facet separation at the site of injury which suggested sufficient stability of the posterior ligamentous structures. Accordingly, we fused $\mathrm{C} 4$ through $\mathrm{C} 6$ anteriorly with an iliac crest bone graft in place of the $\mathrm{C} 5 \mathrm{VB}$ and achieved good postoperative stability (Figure $3 \mathrm{~A}, \mathrm{~B}$ ). Nonunion was observed at 5 months at C5-6 (Figure $3 \mathrm{C}$ ) which was treated with bilateral posterior fusion (Figure $3 \mathrm{D}$ ).
We report the case of a young athlete who was very fortunate to not have sustained permanent neurological sequelae due to cervical teardrop fracture. While hypotheses exist regarding the etiology of the specific teardrop fracture patterns observed clinically, we are unaware of previous biomechanical models that have been able to consistently produce clinicallyrelevant teardrop fractures of the cervical spine. ${ }^{23}$ Case studies which describe mechanisms of teardrop fracture due to sports impacts may help guide and inform future biomechanical investigations for creating realistic injures. These data may ultimately lead to: increased awareness of the injury among athletes, coaches, trainers, and referees; improved training techniques; rule modifications; and design of safer protective equipment and athletic facilities. The data may also provide clinical guidance and information when: choosing optimal patient position intraoperatively and during transport, performing reduction and alignment, and choosing the most appropriate internal or external fixation.

\section{References}

1. Harris JH, Jr. Acute injuries of the spine. Semin Roentgenol 1978;13:53-68.

2. Torg JS, Pavlov H, O'Neill MJ, et al. The axial load teardrop fracture. A biomechanical, clinical and roentgenographic analysis. Am J Sports Med 1991;19:355-64.

3. Richman S, Friedman RL. Vertical fracture of cervical vertebral bodies. Radiology 1954;62:536-43.

4. Lee C, Kim KS, Rogers LF. Triangular cervical vertebral body fractures: diagnostic significance.

AJR Am J Roentgenol 1982;138:1123-32.

5. Kim HJ, Lee KY, Kim WC. Treatment outcome of cervical tear drop fracture. Asian Spine $J$ 2009;3:73-9.

6. Kim KS, Chen HH, Russell EJ, et al. Flexion teardrop fracture of the cervical spine: radiographic characteristics. AJR Am J Roentgenol 1989;152:319-26.

7. Scher AT. 'Tear-drop' fractures of the cervical spine - radiological features. $S$ Afr Med $J$ 1982;61:355-6.

8. Tator CH, Edmonds VE. National survey of spinal injuries in hockey players. Can Med Assoc J 
1984;130:875-80.

9. Tator CH, Ekong CE, Rowed DW, et al. Spinal injuries due to hockey. Can J Neurol Sci

1984;11:34-41.

10. Warwick R, Williams PL eds. Gray's anatomy, 35th British Edition, W.B. Saunders Co. Philadelphia, PAed, 1973.

11. Louis-Ugbo J, Pedlow Jr. FX, Heller JG. Anatomy, biomechanics, and clinical implications. In Benzel EC ed. The cervical spine, 5th Ed., Lippincott, Williams, and Wilkens, Philadelphia, PA, 2012.

12. Kazarian L, Shively R. Biomechanical, radiographic and osteologic observations of lower cervical spine injuries. In Gilchrist MD ed. IUTAM Symposium on Impact Biomechanics: From Fundamental Insights to Applications. University College Dublin, 2005.

13. Clausen JD, Goel VK, Traynelis VC, et al. Uncinate processes and Luschka joints influence the biomechanics of the cervical spine: quantification using a finite element model of the C5-C6 segment. J Orthop Res 1997;15:342-7.

14. Stimpfl A. Zum Problem der sagittalen Langsbriiche der Halswirbelkorper. Chirurg 1949;20:473-74.

15. Harris RS, Jones DM. The arterial supply to the adult cervical vertebral bodies. J Bone Joint Surg Br 1956;38-B:922-7.

16. Carter JW, Mirza SK, Tencer AF, et al. Canal geometry changes associated with axial compressive cervical spine fracture. Spine (Phila Pa 1976) 2000;25:46-54.

17. Ivancic PC, Pearson AM, Tominaga $Y$, et al. Mechanism of cervical spinal cord injury during bilateral facet dislocation. Spine (Phila Pa 1976) 2007;32:2467-73.

18. Ivancic PC. Biomechanics of sports-induced axial-compression injuries of the neck. $J$ Athl Train 2012;47:489-97.

19. Lee C, Kim KS, Rogers LF. Sagittal fracture of the cervical vertebral body. AJR Am J Roentgenol 1982;139:55-60.

20. Panjabi MM, Duranceau J, Goel V, et al. Cervical human vertebrae. Quantitative three-dimensional anatomy of the middle and lower regions. Spine 1991;16:861-9.

21. Jefferson G. Remarks on fractures of the first cervical vertebra. $\mathrm{Br}$ Med $J$ 1927;2:153-7.

22. Jefferson G. Fracture of the atlas vertebra: report of four cases, and a review of those previously recorded. Br J Surg 1920;7:407-22.

23. Alem NM, Nusholtz GS, Melvin JW. Head and neck response to axial impacts. Society of Automotive Engineers Paper No. 8416671984.

\section{Disclosures}

James Yue has consulted for DePuy Spine. The other authors declare no conflicts of interest.

\section{Corresponding Author}

Paul C. Ivancic, Ph.D. Biomechanics Research Laboratory Department of Orthopaedics and Rehabilitation Yale University School of Medicine 333 Cedar St. P.O. Box 208071 New Haven CT 06520-8071, USA.paul.ivancic@yale.edu.

Published 3 February 2016. This manuscript is generously published free of charge by ISASS, the International Society for the Advancement of Spine Surgery. Copyright $\odot 2016$ ISASS. To see more or order reprints or permissions, see http://ijssurgery.com. 